\title{
BNCI Systems as a potential Assistive Technology: ethical issues and participatory research in the BrainAble Project
}

\author{
CLARE CARMICHAEL ${ }^{1}$ and PATRICK CARMICHAEL ${ }^{2}$
}

1. Abilitynet UK, IBM Campus, Birmingham Road, Warwick CV34 5JL and Liverpool John Moores University, Barkhill Road, Liverpool, L17 6BD, UK.

2. Faculty of Education and Sport, Polhill Avenue, University of Bedfordshire, Bedford, MK41 9EA

Correspondence: Clare Carmichael, c/o Abilitynet UK, IBM Campus, Birmingham Road, Warwick CV34 5JL Email: cecarmichael@btinternet.com

\begin{abstract}
Purpose. This paper highlights aspects of the current state of research and thinking about ethical issues in relation to Brain Computer Interface (BCI) and Brain-Neuronal Computer Interfaces (BNCI) research through the experience of one particular project, BrainAble, which is exploring and developing the potential of these technologies to enable people with complex disabilities to control a computer. It describes how ethical practice has been developed both within the multidisciplinary research team and with participants.

Results. The paper presents findings from the project in which participants shared their views of the project prototypes, of the potential of BCI/BNCI systems as an assistive technology, and of their other possible applications. This draws attention to the importance of ethical practice in projects where high expectations of technologies, and representations of 'ideal types' of disabled users may reinforce stereotypes or drown out participant 'voices'.

Conclusions. Ethical frameworks for research and development in emergent areas such as $\mathrm{BCI} / \mathrm{BNCI}$ systems should be based on broad notions of a 'duty of care' while being sufficiently flexible to allow researchers to adapt project procedures according to participant needs. They need to be frequently revisited, not only in the light of experience, but also to make sure that they reflect new research findings and ever more complex and powerful technologies.
\end{abstract}

\section{Implications for Rehabilitation}

- $\mathrm{BCI} / \mathrm{BNCI}$ systems are not similar to existing switch-controlled or eye gaze systems. Users and those supporting them need to have their expectations carefully managed.

- $\mathrm{BCI} / \mathrm{BNCI}$ are emergent technologies and side effects of long term use are not well understood: this demands an ongoing concern to ensure duty of care and maintenance of a 'watching brief' regarding ethical issues.

- Practitioners need to be particularly careful when introducing BCI/BNCI systems to be sensitive to the meanings that are attached to them and how they may convey prognosis.

Keywords: Assistive Technology; Brain Computer Interfaces; Brain-Neuronal Computer Interfaces; Research Ethics; Participatory Research. 


\section{Introduction}

A Brain Computer Interface (BCI) is a system that enables interaction with a computer, based on changing electrical signals that occur in the brain. The signals can be taken invasively (from inside the brain) or non-invasively (from the scalp). Non-invasive BCI systems take signals that are present at microvolt levels on the scalp and then amplify and digitise them so that they can be used by the computer. In a typical BCI setting, participants are presented with stimuli or asked to undertake thinking strategies so that distinctive patterns of electrical activity are generated. Specific signals are extracted and feedback is provided to the user, for example by on-screen activity or through a peripheral device [1]. BCI systems have been identified as having potential as an assistive technology as they make it possible to perform basic commands using thinking strategies alone and they are not reliant on voluntary muscular movement, making them suitable for people with severe communication difficulties [2-4] and in neurological rehabilitation [5] and enabling greater social inclusion [6]. While the effectiveness of these systems is recognised as varying widely, with user motivation being a key determinant of success [7], results have been sufficiently promising [8-10] to warrant further exploration with different groups of users. However, enthusiasm for BCI-based assistive technology based on successful use by non-disabled users has not been fully realised, a recent review stating that combinations of technological and economic constraints mean that "optimism in relation to applications for healthy users is more appropriate than in relation to BCI-based assistive technology" [11], and other authors have suggested that "non-medical" applications of BCI systems such as user state monitoring and gaming may hold more promise [12]. 


\section{Hybrid BNCI Systems and the BrainAble Project}

Current non-invasive BCI systems differ in how they record the neural activity of the brain and in how signals are translated into device commands. Concerns about high levels of 'BCI Illiteracy' leading to the unpredictable individual levels of performance (about $20 \%$ of users fail to successfully use any given BCI system) [13] has led to interest in hybrid Brain Computer Interfaces which use combinations of input signals [14].

BrainAble is an international research and development project funded under the European 7th Framework ICT-2009 Call 4 (Accessible and Assistive ICT); BCI research is highly funded under EU frameworks compared with many other technologies: Euro 34 Million in the current 7th Framework (2007-2013) [15].

BrainAble is unique in that it is a hybrid system and uses different BCI signals in an effort to enable people with complex disabilities to communicate, control environmental systems and ultimately achieve greater levels of independence in everyday living, its broad aims being couched in terms of social inclusion and participation in online and realworld activities. It uses three types of brain activity: sensorimotor rhythms (SMRs), which are associated with imagined movement; steady state visual evoked potentials (SSVEP); and P300 signals. Approaches based on SSVEP and P300 signals require the user to focus their attention on a target that either oscillates or flashes along with other objects with different signals resulting according to whether the object is attended to or ignored. This is the basis of a range of interfaces including environmental control consoles, text-entry systems, navigation systems for virtual reality environments and those for the control of peripheral devices such as robots [16].

Using all three sets of signals improves the reliability of the device and work at the TUGraz BCI laboratory has demonstrated that if one signal is unreliable it was possible to 
switch to another, significantly reducing the problem of BCI illiteracy [17]. Further work is necessary to understand the causes of BCI illiteracy and to explore the best combinations of EEG signals to use in helping users achieve desired goals and implement reliable systems. Brainable is also technically a Brain-Neuronal Computer Interface (BNCI) system. BNCI's integrate other types of biometric signals such as heartbeat, EOG (Electrooculogram) and EMG (Electromyography). The term BNCI is used to refer to the combination of all these different technologies and the BCI signals. The additional biometric signals are used for indirect interaction and can potentially provide indications of boredom, fatigue or frustration. The system developed by the BrainAble project is intended to be 'context-aware' (predicting users' interactions based on previous patterns and offering the most likely options on screen) [18] as well as providing real time feedback to the user regarding success and failure but to also adapt to the user's cognitive state: for example if there is repeated failure and resulting frustration. Self adaptive tools such as fatigue and spasticity control switches whilst in an early stage of development are being tested to see the value and application of providing users with opportunities to change tasks, have instructions repeated and to effectively 'pause' the system.

It is against a background of rapidly evolving technologies; high expectations on the part of funders, the public and potential users; and questions about what directions BCI and BNCI research and development should take, that the BrainAble project took place. The project used participatory design approaches and iterative cycles of user testing and evaluation of the BrainAble prototype in the UK: this was managed by AbilityNet UK and took place at Liverpool John Moores University, who provided a dedicated and accessible site in which the system could be set up and testing conducted. A group of ten non-disabled participants and another group of eight disabled participants with motor disabilities, neurological conditions, and acquired and traumatic brain injuries were 
involved in a total of three iterations of testing and evaluation. This process had two elements: one focused on developing understandings of the individual users' needs and the ways in which the BCI system might support them; and a second relating their experiences to wider populations, informing further development, and assessing the potential of such systems to support different groups of users. This work was also concerned to feed into the broader research agenda about the nature and distribution of 'BCI Illiteracy' and to explore whether, as some research as suggested, it is more common among people with severe disabilities than in the general population and therefore might limit BCI or BNCI systems' potential as assistive technologies.

The project was required to develop a framework for ethical practice, and it is this that is the focus of the remainder of this paper, which describes how this was developed and implemented in the UK-based research, for which NHS National Research Ethics Service consent was given. Later versions of the BrainAble prototype were also tested at the Guttmann Institute in Barcelona, Spain: here, established ethical guidelines based on practice in Spain and already in place at the Institute provided the basis of their work.

\section{Ethical Issues and BCI Systems}

The development and growing public awareness of and interest in BCI research has contributed to a neuro-ethical debate that extends beyond the specifics of particular BCI systems to encompass more wide-ranging discussions about "personhood", mind-reading technologies and polygraphs, surveillance and privacy, the determination of cognitive functioning and 'locked-in' syndrome, and decisions about the termination of life-support systems [19-21]. At the same time, media attention has raised expectations of the potentially transformative and liberating potential of BCI for people with quadriplegia or who are in the advanced stages of progressive conditions such as ALS/MND, leading for 
calls for researchers to focus on developing better relationships with the media and fostering more accurate reporting of progress and prospects [22]. It is against this background it has been argued that that attention needs to be paid not only to the immediate concerns of researchers or participants as BCI systems are developed, but that their broader impact on decision-making on the part of patients and carers, together with longer-term quality-of-life issues, needs also to be considered [23].

The BrainAble project's ethical framework drew upon this work, as well as conforming to the WMA Helsinki Declaration [24] and other ethical guidance produced to aid researchers in ICT projects such as the EU's own ETICA and EGAIS projects [25; 26] and the general funding programme ethical guidelines on 'sensitive areas' of which 'implants and wearable computing' is one [27]. A subsequent review by the Future BNCI project of the programme of which BrainAble is a part has generated further guidelines drawing on more recent BCI and BNCI research and development [15].

As well as the research literatures, EU guidelines, and more general issues that were raised by virtue of the research taking place under UK National Health Service (NHS) research governance, the ethical framework of the project also drew on the experience of assistive technologists (AbilityNet uses the term 'assessor-consultants'). This role involves maintaining an awareness of product developments and research but also a wideranging practice-knowledge, which draws on repertoire of technological expertise as well as a sensitivity to the needs and preferences of people with disabilities [28]. The ethics framework that was developed, then, also reflected this practical experience of working with participants who might be vulnerable or be prone to emotional lability as an aspect of a progressive condition. It also recognised the importance of 'timely' introduction of assistive technologies [29] and the need to avoid accidentally or insensitively conveying 
prognosis by drawing attention to features of the BCI system through observations along the lines of "you might not need this yet but ...".

\section{Developing the BrainAble Project Ethical Framework}

The ethical framework was articulated, for the purposes of submission through the Integrated Research Application System (IRAS) for review by a specialist NHS Research Ethics Committee (REC), as a research protocol, site-specific information about location and nature of the testing process, and a complete set of supporting documentation including copies of all the research instruments, surveys and questionnaires; inclusion and exclusion criteria; consent forms; and participant information documents, leaflets and recruitment posters. The REC selected to scrutinise the application was one whose members had experience both of work with participants considered 'vulnerable' and that involving medical devices.

The various documents presented synthesised information from a wide range of sources and, particularly in the case of recruitment and information documents, deliberately addressed issues raised by then-current media coverage of BCI systems. This synthesis is exemplified in the following extracts, which give an indication of how the project was presented both to the Research Ethics Committee and, subsequently, to potential participants.

The issues of how participant wellbeing would be safeguarded and the avoidance of any emotional distress were discussed, particularly given the potential role of participation in provoking participants to re-examine their physical or cognitive capacities or to become aware of the progress of conditions, an issue highlighted by Pape et al. [30] and reflected in the submission as follows: 
"The participants in our study may be severely disabled people and will not be able to comfortably manage long sessions. At all times, users wishes will be observed with regard to testing periods of time and frequency so may be subject to changes based on their individual circumstances. An extended series of interviews and testing also allows opportunities to explore existing patterns of technology use and to allow reflective evaluation on the part of the participants" (Submission to Research Ethics Committee)

"We wish to ascertain what meanings the prototype has for people but with sensitivity and not by causing them emotional discomfort. There are important issues around conveying prognosis for people with progressive conditions and especially with MND, by discussing a prototype that has widespread literature that discusses the potential course of the condition and the potential for 'locked in' syndrome ... This is one of the key reasons for the researcher spending time with participants ahead of the introduction of the technology in a 'test' situation, rather than carelessly offering it and discussing future limitations of which the participant may be unaware ..." (Submission to Research Ethics Committee)

General misconceptions about the nature of BCI systems and their potential were also identified in the submission and also addressed in participant information:

"Some initial experiences have shown that people's understandings of BCI devices are hazy at best. Expectations from science fiction films and overenthusiastic media coverage need to be tempered both in terms of managing expectations and at the same time reassuring participants this BCI system is not a mindreading device or lie detector." (Submission to Research Ethics Committee)

"It can be quite exciting to carry out the activities successfully using the BCI as you are controlling your environment seemingly by thinking. However the BCI is not in any way a 'mind reading' device!' (Participant Information Document)

The state of development of the BrainAble system in particular was also made clear: 
"A prototype is an early model to test a concept and is something to be learned from; it is not a finished product. The BrainAble prototype has been put together by our research teams ... It is a working prototype that includes a groundbreaking but existing safety marked Brain Computer Interface with a P300 system which includes the electrode cap and amplifier. This is being used in a new way to control a computer with software that we are testing. This controls smart home, virtual reality and communication components." (Participant Information Document)

Information sheets, flyers and recruitment posters all illustrated the system being used so that participants were aware of the appearance of the components, the fact that gel electrodes were used, and that the computer system was complex and not an 'off the shelf' system.

The prototypical nature of the system and the tentative claims that were made about how close to market the prototype was were also highlighted in relation to the research approaches to be used:

"[The project] includes a marketing strategy designed to ensure it is the best option for users, rather than being driven by enthusiasm, media interest or high profile cases. Investigating the 'fit' of the BCI and critically evaluating how it compares with other options (including those already in use by user) is therefore vitally important and has been included in the design of this research and the use of existing instruments such as the PIADS Form and the MPT." (Submission to Research Ethics Committee)

Following on from this, and recognising that use of diagnostic tools such as PIADS and MPT might generate other insights and awareness (on the part of both researchers and participants), the submission continued:

"In the course of this research, it is likely that we may come across potential participants who would like help with their existing Assistive Technology. In 
this case we have permission to refer them to the AbilityNet call centre, to the fact sheets that AbilityNet provide, and to other appropriate agencies."

(Submission to Research Ethics Committee)

The submission also addressed two key areas related to the nature of participants' disabilities, and the resulting claims that might be made about the potential role and impact of the BCI system. First, it highlighted the importance of being clear about what was meant by terms like 'locked in', given recent media coverage of BCI systems:

"Locked-in Syndrome is ... a rare neurological disorder characterized by complete paralysis of voluntary muscles in all parts of the body except for those that control eye movement ... Individuals with Locked-in Syndrome are conscious and can think and reason, but are unable to speak or move. The disorder leaves individuals completely mute and paralyzed. At present the study does not include people who are strictly defined as 'locked in' but this is a consideration and the BCI is offering hope to people concerned about a locked in state." (Submission to Research Ethics Committee)

The second area where misconceptions existed about medical conditions and disability, both in general and amongst BCI researchers, concerned the cognitive abilities of people with ALS/MND. While ALS/MND patients, even those in a 'Locked-in' state (in the strict sense) have widely been regarded as being cognitively intact, although they have lost the ability to communicate by traditional means, this has been challenged $[31 ; 32]$ and ALS/MND is increasingly being seen as a "complex multi-systems disorder" [33]. The submission argued that the fact of a spectrum of cognitive impairment, and associated lack of motivation or attention, might make it difficult to make meaningful judgements about the performance of the BCI system [34] or, for that matter, about its suitability as a long-term AT solution, and highlighted the relevant research:

"This is significant for our research in several further respects. BCI studies of ALS patients who had not been screened [for Cognitive Impairment] report 
variability in response and signal strength. Sellers et al. (2003) report that the patients found it difficult to communicate by spelling text character-bycharacter ... Also significant for this study is Strong's (2008) study of the impact of [Frontotemporal Lobar Degeneration] in the course of ALS, both its outcomes and the potential impact on the quality of life not only the patient but also for the caregiver." (Submission to Research Ethics Committee)

The experience of completing such a detailed and rigorous ethical submission and addressing the questions and issues it raised was very valuable and led to clearer articulations of methodological issues and research relationships on the part of project researchers. NHS ethical approval processes understood and categorised the project, the BCI system and BCI research in general. As far as the REC was concerned, participants with disabilities were 'vulnerable' (regardless of their self-assessment) and a broad duty of care on the part of researchers was more important than obtaining ethical 'clearance'. And however much advocates of BCI systems might want to present them as new (noninvasive) interfaces, input devices, or assistive technologies, the involvement of the EEG cap and the collection of brain activity and other participant data firmly located the BCI system as a 'medical device' and this determined the standards of ethical practice to which the project should conform.

The REC responses to the specific research plans of project raised a number of issues about working with emergent technologies about which there was public interest and that care should be taken to reflect the newness of the field, the fact that the system was combining a number of elements for the first time (possibly with unpredictable outcomes) and that participants should be made very aware that what was being tested was a prototype that most likely would have few tangible benefits to them as individuals. In particular, they highlighted the issue 'BCI Illiteracy' and recommended that participants' expectations be managed, and support provided, in relation to this: 
"The committee expressed concern over the statistic that you expect that up to $10 \%$ of participants will not be able to 'train their brains' to use the device and will be left disappointed following their participation in the study, particularly the disabled participants, and wanted clarification that there would be someone available to talk them through their disappointment and discuss any options with them.” (REC Committee Ethical Opinion Statement)

It was confirmed that someone would be available as suggested, and they were satisfied that this tasks of managing expectations prior to participation, and supporting participants afterwards, could be undertaken by an experienced Assistive Technology Assessor-Consultant.

\section{Ethical Issues in Practice}

Once the testing of the BNCI system began with participants, some of the issues raised in research and practice and included in the ethical submission did indeed manifest themselves and were reflected in the way that the actual testing was conducted.

In response to the REC requirement that the prototypical nature of the BrainAble system was made clear, this was further elaborated in the Participant Information and this helped participants and researchers in managing expectations. This proved useful during the extended periods needed for setup and calibration, and on those occasions when the equipment or software did not work reliably. The system was set up with not only user interfaces but also programming elements and the developer environment clearly visible. Whilst there was a monitor for participant use they were also nearly all interested in the researcher interface as well, although towards the end of the study one participant observed that he would not want to see the programming elements in any end product. All participants were very patient when computers needed rebooting and offered to continue beyond points that seemed reasonable junctures at which to suspend efforts. 
At the same time, the prototypical nature of the system meant that there was a reluctance on the part of many participants to voice any real criticisms and that the researcher often needed to make inferences and then suggest these to solicit participants' agreements or disagreements. One participant was with his parents on one occasion and their response to the system had been that "it's marvellous for him take part in such a study". They also requested a record of the study as they kept a scrapbook for their son and it was touching that, as well as more general project information, they wanted a personalised record of his participation. But, when they were asked (in an inferential, 'what-if' way) how they would feel if they were offered such a system for personal use, his mother's reaction was clear, and she exclaimed "What, with that cap?" When questioned further, she finally offered that she didn't like the "medical" nature of the EEG equipment but that if it did offer him some independence then its advantages might outweigh this. However, as it stood, the aesthetics of the EEG cap and the medical connotations it carried were, in her view, a significant barrier to adoption.

Other participants also discussed the EEG cap: all participants wanted to see how they looked with it on and mirrors were provided for this purpose: some also wanted to see photographs of themselves wearing the cap. For some it generated some nervous laughter and in the case of two friends who came together some hilarity; something like a "baseball cap" was felt to be preferable, not only for aesthetic purposes but, again, because of the medical connotations of the electrode-laden EEG cap, the complexity of which was identified as an area of concern by several of the participants' carers. The dependence of the cap on gel electrodes was also an issue for some participants, and for females in particular, who proved difficult to recruit, possibly as a result: most of the participants had wanted to see and touch the gel at the recruitment interview stage, and its use may have been a reason for their lack of engagement. One female participant brought 
with her to a testing session a beret, which she said she'd brought to wear afterwards, although in the event, she didn't feel she needed it as the gel had brushed out. We can conclude from this that it was important to inform potential participants about nature of the cap and the use of the gel, and to specifically mention it in the consent form.

Participant responses to the prototypical nature of the system did, however, bring about a significant decision about the conduct of the testing process. After a hardware failure during a test session with a non-disabled but expert participant (who had a good knowledge of the issues around ALS/MND), they expressed the view that involving participants in working with a prototype was acceptable, but that "people should feel part of something that worked and which was taking things forward". These concerns were conveyed to the wider project team and after some discussion of these events, the particular iteration of testing (the first) was concluded so that issues of build quality and reliability could be addressed. The EU programme reviewers ratified this decision as appropriate on methodological and ethical grounds when the project made its annual reports.

As had been expected, 'BCI Illiteracy' proved to be an issue: one participant was unable to use the system despite multiple attempts across test sessions. Test session data were sent to project partners for analysis, and use of other input methods revealed that this particular pattern of 'Illiteracy' was associated with reduced information processing speed, an aspect of the participant's underlying condition [35]. The participant was disappointed and suggested that he was wasting project time and resources, but was reassured that his contribution was both valid and valued, not only by the UK-based researchers but also by project partners when they visited Liverpool. Most importantly, the participant continued to be involved in the project, offered feedback and evaluation of 
aspects of the BCI system and was not excluded on the grounds that he had been unable to use the BCI consistently or reliably.

Another area highlighted in the ethics submission was that of recruitment, reimbursement and obtaining informed consent from participants. Disabled participants had travel expenses reimbursed for expenses and were given an additional payment recompense for their time. Some expressed a view that this was unnecessary and were initially vague about whether they wanted to be paid or how best this could be achieve, but as testing progressed, it became clear that they all did want payment. This suggests that in studies involving disabled participants processes and procedures should be set out clearly from the outset, and participants should not be placed in potentially difficult or embarrassing positions in relation to remuneration. Interestingly, the approach adopted by BrainAble differs from that in some other BCI/BNCI projects, where it is non-disabled participants who are paid and disabled ones not, on the ground that the latter are potential beneficiaries of the research.

Rather in contrast, obtaining informed consent proved less problematic, although a number of strategies were developed in order to answer questions raised by participants, to obtain and revisit consent (particularly in the cases of participants who had short-term memory loss following traumatic brain injury), and to make sure that views on the system were adequately expressed. Some of participants could be described as severely disabled, so obtaining informed consent was not straightforward. In the submission to the REC it had been suggested that videoing consent could be an option to help ensure consent was given, whereby an independent witness could watch the process on video and verify that they felt that consent had been expressed; however this method was not in fact used and instead we involved carers and witnesses. An independent witness watched the process on 
video and verified that they felt that consent had been expressed; when additional witnesses and carers (many of whom were used to communicating with the disabled participants) were present, they were also asked to confirm that they felt informed consent had been given. In one instance, where a severely disabled participant was testing the system, there was also a prior case conference and close relatives as well as caseworkers were also asked to provide consent. It was agreed that he should be involved; he was able to use the BCI device; and once engaged, regular pauses and questions determined through his limited and inconsistent voluntary movement, that he wished continue to test the system.

One of the participants with AAC needs did suggest that being able to ask for additional information on certain aspects of the study and the system would be useful. The BrainAble project researcher then explored the idea of using "Speakbook", a low-cost, low-tech communication tool that works by following the direction of gaze at a board [36]. Working together, the researcher and participant were able to identify some of the questions that other participants might wish to ask, so that the researcher could elaborate. This could also be used in the future to help with informed consent where, while participants have little muscular control or speech, there is a discernible gaze direction. The key point that this process demonstrated was that, rather than simply replicating informed consent documents in new forms, things like "SpeakBook" work best when expert participants with insights into the concerns of disabled people have a role in their design.

While each of the forgoing issues had been identified (in broad terms, if not in detail) in the ethical framework and submission, unexpected issues also emerged, and it was here 
that broad ethical principles had to be enacted in practice as the project proceeded with its work.

The ethics submission had suggested that, should the need arise, participants could be referred for an assessment by AbilityNet. In fact, this did not happen, although participation in the project enabled participants to improve their awareness of Assistive Technologies and computer systems in general: one adopting "Speakbooks" and another setting up their home computer with multiple monitors and enlarged text. In another example, a participant had increasing communication difficulties but did not currently have a computer system that he wanted to use. Here, the potential for an assessment or intervention seemed more obvious, and as he was able to use the P300-based system accurately and comparatively rapidly, one of the commercial partners in the BrainAble Consortium offered a BCI system for the participant for his own use at no cost. This was offered informally to the participant through an intermediary, but was not accepted: the participant's responses were based on concerns that the system was still in too early a stage of development to be something he could use confidently and independently; that its high cost and perceived 'cutting edge' nature would make it an insurance risk; and, following from both these, that he would prefer something more 'mainstream' and widely used. The informality and discretion with which the offer was made was well-advised: the participant was in the process of trying to obtain funding for Assistive Technology and the appearance of a high-cost system from a 'third party' supplier might well have compromised this funding or other support. Again, as Dobkin argues, the ethical scope of decisions made about particular technological systems may extend far beyond the research site and impinge on the lives and life-chances of patients and carers [23]. 
The UK-based user testing, from the outset, had been concerned with exploring and developing the potential of the BCI system as an Assistive Technology, and much of the data collection was oriented towards this aim. However, in the course of testing, the emergent nature of the technologies meant that the research took unexpected trajectories and generated outcomes that had not been predicted - either in the overall project design or the ethical framework. The potential of the system to identify information processing issues has already been mentioned; but with another participant, engagement with the BCI system and the testing processes associated with it, led not only to insights into their individual condition and needs, but also offered a pointer to an alternative role for BCI systems more generally. This participant had suffered a traumatic brain injury and had recently moved into a rehabilitation setting where his needs were being defined, and rehabilitation and care plans drawn up. Set against a background of uncertainty about his level of cognitive functioning, successful use of the BrainAble prototype provided information that was shared beyond the project and informed these plans.

This example highlights how important it is for researchers to recognise that, because a technology is emergent and prototypical, its role may not ultimately be what we predict and the relationships established with participants may lead in entirely different directions. The two examples offered here both challenged the premises of the BrainAble project (that BCI systems could act as Assistive Technologies and that disabled people would welcome them) and raise questions about how to be responsive to such unpredictability while still behaving ethically. When technologies are emergent, it is difficult to predict what affordances will be recognised in them, and project-level ethical practices need, in these situations, to be grounded in broader ethical frameworks and commitments. 


\section{Conclusions}

The original BrainAble project proposal to its funders conveyed the potential of BCI systems through the presentation of a 'persona': an ideal user who was a young female student who had a motorbike accident and was consequently paralysed. Had the project then been construed as largely (or even entirely) concerned with technical development, there would have been a danger that ever more sophisticated BCI systems and peripherals would have emerged, still premised on plausible fictions and heroic narratives of transformation and independence. Were this to be the case, the danger is that methodologically and ethically significant issues emerging from research (such as potential side effects of prolonged use of BCI systems, or of the possibility that people with ALS/MND are not all cognitively unimpaired) would be drowned out by popular media hype and enthusiasm.

By conceptualising and enacting the testing of the Brainable system as an iterative and participatory process in which disabled participants are involved as "active empowered partners" [37], they have been far more than test subjects. Their voices have contributed to the direction of the project and the authentic accounts of their lives, the role that Assistive Technologies and BCI systems might play, and the meanings that they might convey, have introduced ethical issues into the cross-disciplinary debates that are inherent in a project of this kind. Rather than seeing participants as representatives of ideal user types, aggregating data collected from them and anonymising their contributions, it was evident across our participant group that they were both keen to have their stories told, have their participation acknowledged and to act as representatives and advocates.

As we have demonstrated, ethical issues were identified by the literature on BCI systems; by Assistive Technologists; NHS Ethicists; and participants themselves varied. 
Furthermore, the rapidly developing nature of the field and the as yet unclear role of BCI systems threw up further challenges. This has demanded open and continuous communication between the different members of the multi-disciplinary project team: with user data from research settings and from the field more widely being shared with technology developers, and technological features of new prototypes being reflected in ethically sound implementation and evaluation.

In short, new technologies, and the ethical practices and discourses that accompany them need to be seen as co-constituted, rather than with ethical practice subordinated to technological development and deployment, or financial imperatives. Ethical frameworks and practices in general need to reflect rapidly changing technologies, not only so that users can be offered the best possible experience, but also so that new research about potential side effects or impacts of participation can be reflected in research designs, and incorporated into participant information and informed consent arrangements.

Central to this information exchange and development of practice are the voices of disabled people. This article represents not only a contribution to debates about the importance of those voices, but has tried, itself, to give voice to participants and contributors - not only to a process of technological development and evaluation, but to the ethical practice too. We are sharing these as part of a programme of ethical practice we hope continues to develop.

\section{Acknowledgements and Declarations}

The BrainAble Project (Project No. 247447 'Brainable: Autonomy and social inclusion through mixed reality Brain-Computer Interfaces: Connecting the disabled to their physical and social world') is funded under the European Commission's $7^{\text {th }}$ Framework Programme as part of the ICT-2009 Call 4 “Accessible and Assistive ICT”. Project 
partners are: Barcelona Digital Centre Technologic (Spain); TU Graz (Austria); Universitat Pompeu Fabra (Spain); Meticube Software (Portugal); Guger Technologies (Austria); Abilityet (UK) and Institut Guttmann (Spain). Full details are at the project website: http://www.brainable.org. The contribution of all other project partners is acknowledged, as is the support of Liverpool John Moores University who provided project premises and site-specific governance, and with which both authors were affiliated during the ethical review process and early iterations of project testing.

There are no conflicts of interest in relation to the publication of this work, and all data presented here as been collected in accordance with the project ethical code of practice. 


\section{References}

1. Wolpaw, JR. 2007. Brain-computer interfaces as new brain output pathways. J Physiol 579:613-619.

2. Wolpaw, JR, Birbaumer, N, McFarland, DJ, Pfurtscheller, G, Vaughan, TM. 2002. Brain-computer interfaces for communication and control. Clinical Neurophysiology 113:767-791.

3. Cincotti, F, Mattia, D, Aloise, F, Bufalari, S, Schalk, G, Oriolo, G, Cherubini, A, Marciani, MG, Babiloni, F. 2008. Non-invasive brain-computer interface system: towards its application as assistive technology. Brain Res Bull 75:796-803.

4. Pfurtscheller, G, Muller-Putz, GR, Scherer, R, Neuper, C. 2008. Rehabilitation with Brain-Computer Interface Systems. Computer 41:58-65.

5. Daly, JJ, Wolpaw, JR. 2008. Brain-computer interfaces in neurological rehabilitation. The Lancet Neurology 7:1032-1043.

6. McCullagh, PJ, Ware, MP, Lightbody, G. 2010. Brain Computer Interfaces for Inclusion. AH10, 1st International Augmented Human Conference Article 6:1-8 Megeve, France. http://portal.acm.org/citation.cfm?doid=1785455

7. Kleih, SC, Nijboer, F, Halder, S, Kubler, A. 2010. Motivation modulates the P300 amplitude during brain-computer interface use. Clin Neurophysiol 121:1023-1031.

8. Allison, B, Luth, T, Valbuena, D, Teymourian, A, Volosyak, I, Graser, A. 2010. BCI demographics: how many (and what kinds of) people can use an SSVEP BCI? IEEE Trans Neural Syst Rehabil Eng 18:107-116.

9. Guger, C, Daban, S, Sellers, E, Holzner, C, Krausz, G, Carabalona, R, Gramatica, F, Edlinger, G. 2009. How many people are able to control a P300-based braincomputer interface (BCI)? Neurosci Lett 462:94-98.

10. Volosyak, I, Valbuena, D, Luth, T, Malechka, T, Graser, A. 2011. BCI demographics II: how many (and what kinds of) people can use a high-frequency SSVEP BCI? IEEE Trans Neural Syst Rehabil Eng 19:232-239.

11. Nijboer, F, Allison, BZ, Dunne, S, Plass-Oude Bos, D, Nijholt, A, Haselager, P. 2011. A Preliminary Survey on the Perception of Marketability of Brain-Computer Interfaces and Initial Development of a Repository of BCI Companies. 5th International Brain-Computer Interface Conference, BCI 2011 Graz, Austria. http://doc.utwente.nl/78138/

12. van Erp, J, Lotte, F, Tangermann, M. 2012. Brain-Computer Interfaces: Beyond Medical Applications. Computer 45:26-34.

13. Kubler, A, Muller, K-R. 2007. An Introduction to Brain-Computer Interfacing. In: Dornhege, G, Millan, J, Hinterberger, T, McFarland, D, K-R, M, editors. Towards Brain-Computer Interfacing. Cambridge, MA: MIT Press. p. 1-25. 
14. Pfurtscheller, G, Allison, BZ, Brunner, C, Bauernfeind, G, Solis-Escalante, T, Scherer, R, Zander, TO, Mueller-Putz, G, Neuper, C, Birbaumer, N. 2010. The hybrid BCI. Front Neurosci 4:30.

15. Future BNCI. 2012. Future BNCI: A Roadmap for Future Directions in Brain/Neuronal Computer Interaction Research. Graz: Future BNCI Project.

16. Guger, C, Edlinger, G, Krausz, G. 2011. Hardware/Software Components and Applications of BCIs. In: Fazel, R, editor. Recent Advances in Brain-Computer Interface Systems. InTech. p. 1-25.

17. Brunner, C, Allison, BZ, Krusienski, DJ, Kaiser, V, Muller-Putz, GR, Pfurtscheller, G, Neuper, C. 2010. Improved signal processing approaches in an offline simulation of a hybrid brain-computer interface. J Neurosci Methods 188:165-173.

18. Navarro, AA, Ceccaroni, L, Velickovski, F, Torrellas, S, Miralles, F, Allison, BZ, Scherer, R, Faller, J. 2011. Context-Awareness as an Enhancement of BrainComputer Interfaces. Ambient Assisted Living (Lecture Notes in Computer Science 6693). Berlin, Heidelberg: Springer. p. 216-223.

19. Riis, P. 2003. Neuroethics. European Journal of Neurology 10:218-223.

20. Tamburrini, G. 2009. Brain to Computer Communication: Ethical Perspectives on Interaction Models. Neuroethics 2:137-149.

21. Wolpe, PR. 2007. Ethical and Social Challenges of Brain-Computer Interfaces. Virtual Mentor 9:128-131.

22. Haselager, P, Vlek, R, Hill, J, Nijboer, F. 2009. A note on ethical aspects of BCI. Neural Networks 22:1352-1357.

23. Dobkin, BH. 2007. Brain-computer interface technology as a tool to augment plasticity and outcomes for neurological rehabilitation. J Physiol 579:637-642.

24. World Medical Association. 2008. Ethical Principles for Medical Research Involving Human Subjects (Declaration of Helsinki). http://www.wma.net/en/30publications/10policies/b3/17c.pdf

25. ETICA. 2010. ETICA: Ethical Issues of Emerging ICT Applications. EiEX: Journal of the European Innovation Exchange 3.

http://www.eiex.eu/docs/EIEX03ETICA01-05.pdf

26. EGAIS. 2011. EGAIS: The Ethics of Technology. EiEX: Journal of the European Innovation Exchange 7. http://www.eiex.eu/docs/EIEX05EGAIS.pdf

27. CORDIS. 2010. Ethical Guidelines for undertaking ICT research in FP7. $\mathrm{ftp} / / / \mathrm{ftp}$.cordis.europa.eu/pub/fp7/docs/guidelines-annex5ict.pdf

28. Folkes, C, Carmichael, P. 2006. 'Learning to assess' and 'assessing to learn': the construction of knowledge about Assistive Technology. Educational Action Research 14:535-545. 
29. Folkes, C. 2004. Last Voices: An Exploration of the Nature of Assistive Technology Interventions for People With Motor Neurone Disease [MA dissertation]. Reading University.

http://www.sigaccess.org/community/theses_repository/masters/clare_folkes.php.

30. Pape, T, Kim, J, Weiner, B. 2002. The shaping of Individual Meanings Assigned to Assistive Technology: A review of Personal Factors. Disability and Rehabilitation 24:5-20.

31. Hanagasi, HA, Gurvit, IH, Ermutlu, N, Kaptanoglu, G, Karamursel, S, Idrisoglu, HA, Emre, M, Demiralp, T. 2002. Cognitive impairment in amyotrophic lateral sclerosis: evidence from neuropsychological investigation and event-related potentials. Cognitive Brain Research 14:234-244.

32. Lomen-Hoerth, C, Murphy, J, Langmore, S, Kramer, JH, Olney, RK, Miller, B. 2003. Are amyotrophic lateral sclerosis patients cognitively normal? Neurology 60:1094-1097.

33. Strong, MJ. 2008. The syndromes of frontotemporal dysfunction in amyotrophic lateral sclerosis. Amyotroph Lateral Scler 9:323-338.

34. Sellers, E, Schalk, G, Donchin, E. 2003. The P300 as a typing tool: Tests of brain computer interface with an. ALS patient. Psychophysiology 40:s77.

35. Forn, C, Belenguer, A, Parcet-Ibars, MA, Avila, C. 2008. Information-processing speed is the primary deficit underlying the poor performance of multiple sclerosis patients in the Paced Auditory Serial Addition Test (PASAT). J Clin Exp Neuropsychol 30:789-796.

36. Speakbook. 2011. Speakbook: Nonprofit Communication Tools. http://www.speakbook.org/

37. Olsson, E. 2004. What active users and designers contribute in the design process. Interacting with Computers 16:377-401. 
